\title{
Optical shift register based on an optical flip-flop memory with a single active element
}

\author{
S. Zhang, Z. Li, Y. Liu, G. D. Khoe and H. J. S. Dorren \\ COBRA Research Institute, Eindhoven University of Technology, P.O. Box 513,5600 MB, Eindhoven, the Netherlands \\ s.zhang@tue.nl
}

\begin{abstract}
We present an optical shift register that consist out of two serially connected optical flip-flop memories driven by common clock pulses. Each optical flip-flop consists out of two ring lasers sharing a single active element, which makes the optical flip-flops easily cascade with each other. The two cascaded optical flip-flops are controlled by the clock pulses in such a way that the input data set the new state of the first optical flipflop, after the state of the first flip-flop has been transferred to the second optical flip-flop. The concept is demonstrated at an operation speed of 20 $\mathrm{kHz}$, which is limited by the $10 \mathrm{~m}$ long laser cavities formed by the fiber pig-tailed components.
\end{abstract}

(C)2005 Optical Society of America

OCIS codes: (210.4680) Optical memories; (200.4560) Optical data processing.

\section{References and link}

1. N. A. Whitaker Jr., M. C. Gabriel, H. Avramopoulos, and A. Huang, "All-optical, all-fiber circulating shift register with an inverter," Opt. Lett. 16, 1999-2001 (1991).

2. A. J. Poustie, R. J. Manning, and K. J. Blow, "All-optical circulating shift register using a semiconductor optical amplifier in a fibre," Electron. Lett. 32, 1215-1216 (1996).

3. B. Tian, W. V. Etten, and W. Beuwer, "Ultrafast all-optical shift register and its perspective application for optical packet switching," IEEE J. Sel. Top. Quantum Electron. 8, 722-728 (2002).

4. S. Zhang, Y. Liu, D. Lenstra, M. T. Hill, H. Ju, G. D. Khoe, and H. J. S. Dorren, "Ring-laser optical flipflop memory with single active element," IEEE J. Sel. Top. Quantum Electron. 10, 1093-1100 (2004).

5. H. Kawaguchi, "Bistabilities and Nonlinearities in Laser Diodes," (Artech House, London, 1994).

6. M. T. Hill, H. J. S. Dorren, T. J. de Vries, X. J. M. Leijtens, J. H. den Besten, E. Smalbrugge, Y. S. Oei, G. D. Khoe, and M. K. Smit, A fast low-power optical memory based on coupled micro-ring lasers. Nature 432, 206-209 (2004).

7. S. Zhang, D. Owens, Y. Liu, M. T. Hill, D. Lenstra, A. Tzanakaki, G. D. Khoe, and H. J. S. Dorren, Multistate optical memory based on serially interconnected lasers. IEEE Photon. Technol. Lett. 17, 1962-1964 (2005).

\section{Introduction}

Optical shift registers have received considerable attention since they could be potentially applied in optical packet buffers and serial-to-parallel converters [1-3]. Several approaches have been explored. It is shown in Refs. $[1,2]$ that circulating optical shift registers are realized using either fiber buffers or using Sagnac interferometers. Another example, given in Ref. [3], is based on coupled SEEDS (self-electro-optic effect device) driven by changes of the driving voltage.

In this paper, we present an optical shift register based on cascaded optical flip-flop memories driven by common optical clock pluses. The optical flip-flop memory consists of two ring lasers that share a single active element [4], which makes that the optical flip-flops easily cascade with each other. The optical shift register configuration is generic. This concept can be implemented using different optical flip-fops [5]. Ultra-compact optical flip-flops with a switching speed that is potentially greater than $100 \mathrm{GHz}$ have been demonstrated in Ref. [6]. It has been shown that optical flip-flops can be coupled without isolation to form memories [7]. This means that this concept has potential to be integrated and to perform at high speed.

\#7971 - \$15.00 USD

(C) 2005 OSA
Received 29 June 2005; revised 11 November 2005; accepted 11 November 2005

28 November 2005 / Vol. 13, No. 24 / OPTICS EXPRESS 9708 


\section{Operation principle}

Figure 1 shows a block diagram of the optical shift-register, which consists of an optical converter in combination with two cascaded optical flip-flop memories. The optical flip-flop memory consists of two coupled ring lasers [4] which are bi-stable in the sense that in each stable state one of the lasers lases while the other is suppressed. The state of the optical flipflop memory is thus determined by the wavelength of the dominant laser. Therefore, an optical converter is needed to transform optical data encoded by two binary intensity levels (on and off) into data encoded in two different wavelengths corresponding to the two states of the optical flip-flop. Each optical flip-flop has two input ports and two output ports. The light injected into the port 'In1' can not set a new state unless the optical flip-flop is cleared by a strong external optical pulse injected into the port 'In2'. Two optical flip-flops are cascaded by connecting the port 'Out1' of optical flip-flop 1 with the port 'In1' of optical flip-flop2. Port 'Out2' of each optical flip-flop is used to output light for monitoring.

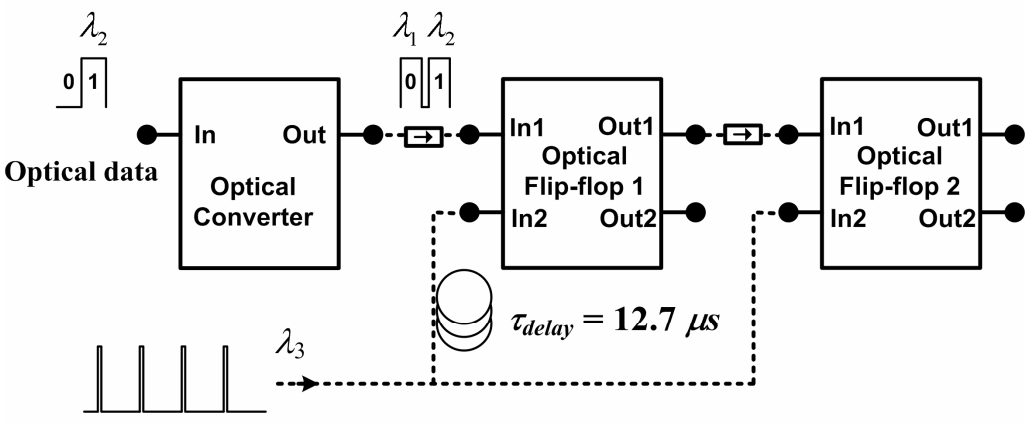

Optical clock pulses

Fig. 1. Schematic configuration of the optical shift register.

The optical shift-register operates in the following fashion. Firstly, the intensity modulated input data are transformed by the optical converter into wavelength encoded data and subsequently injected into the port 'In1' of flip-flop1, which is cascaded with optical flip-flop 2. The two cascaded optical flip-flops are controlled by optical clock pulses that are required to clear the optical flip-flops. We use an external optical clock signal whose power is divided into two parts. 50\% of the power is delayed while the other $50 \%$ of the power is used to clear the state of optical flip-flop 2 (by injection via its port 'In2'). The output from optical flip-flop 1 then sets the new state of optical flip-flop 2. After this operation, the delayed clock pulse is injected into the port 'In2' of optical flip-flop 1 and subsequently clears its state. The signal encoded in wavelength that outputs from the optical converter then sets the new state of optical flip-flop 1 . Thus a compete 'shift' function has been realized.

\section{Experiment results}

The optical shift register shown in Fig. 1 is implemented using fiber pig-tailed components. The optical converter consists of a unidirectional ring laser as shown in Fig. 2(a). A bulk strained semiconductor optical amplifier (SOA) that acts as the active element has a typical small gain of $20 \mathrm{~dB}$ at $200 \mathrm{~mA}$ and a $3 \mathrm{~dB}$ saturation power of $-5 \mathrm{dBm}$. A Fabry-Perot filter (3 $\mathrm{dB}$ bandwidth $0.20 \mathrm{~nm}$ ) with a wavelength of $1550.92 \mathrm{~nm}\left(\lambda_{1}\right)$ is used as a wavelength selective element. An isolator is used to ensure unidirectional lasing. The optical data to be injected into the ring laser are at a wavelength of $1552.52 \mathrm{~nm}\left(\lambda_{2}\right)$ and have an average power of $3 \mathrm{dBm}$, as shown in Fig. 2(c). If the injected information is a binary ' 1 ', $50 \%$ of the injected optical power is directly coupled to the output port. The other half of the injected light is coupled into the ring cavity by a 50/50 coupler and suppresses the lasing by saturating the 
SOA. The light that outputs the optical converter is thus at the wavelength $\lambda_{2}$, as indicated in Fig. 2(d). If no light is injected into the ring laser (a binary ' 0 '), the ring laser remains lasing at the wavelength $\lambda_{1}$, as indicated in Fig. 2(e). In this case, the light that outputs the optical converter is at the wavelength $\lambda_{1}$. Thus the data encoded in intensities are transformed by the optical converter into the data encoded in wavelengths.
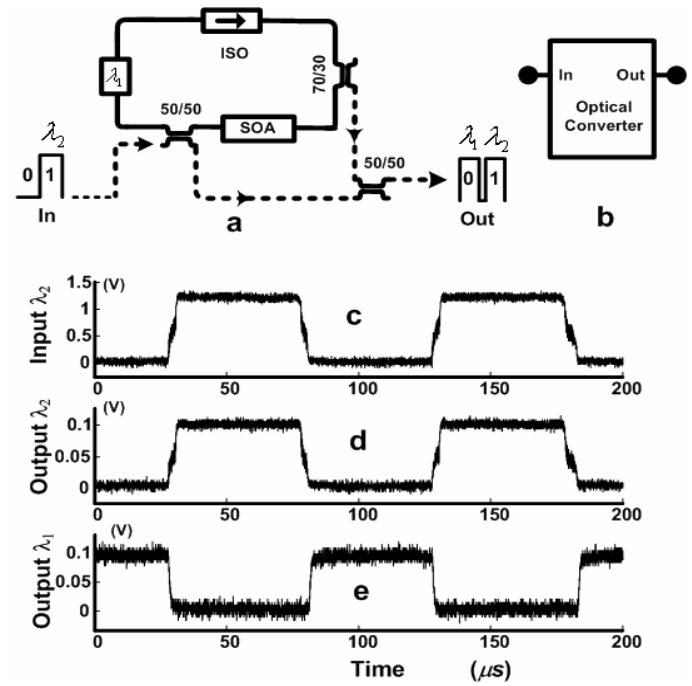

Fig. 2. (a) The configuration of the optical converter. (b) The symbol that we use to indicate the optical converter. (c) The signal injected into the optical converter at the wavelength of $\lambda_{2}$ $(1552.52 \mathrm{~nm})$. (d) and (e) The signal that outputs the optical converter at the wavelengths of $\lambda_{2}$ and $\lambda_{1}(1550.92 \mathrm{~nm})$, respectively. ISO Isolator.
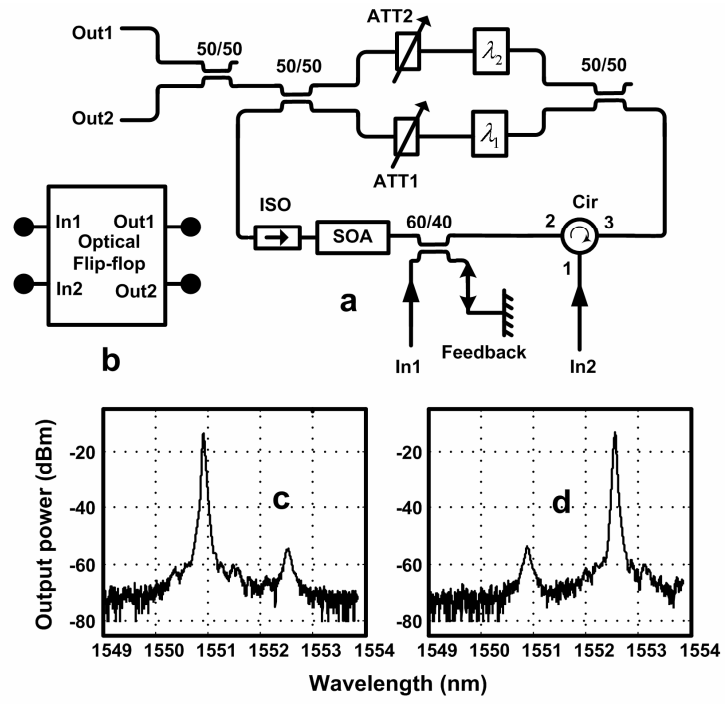

Fig. 3. (a) Schematic configuration of the optical flip-flop memory. (b) The symbol that we use to indicate the optical flip-flop memory. (c) and (d) Experimental results to show that the system has two stable states. ATT: Attenuator

Each optical flip-flop memory consists of two ring lasers sharing a SOA and a feedback arm as shown in Fig. 3(a). The two ring cavities are formed using two 50/50 couplers. Each ring cavity contains a Fabry-Perot filter acting as a wavelength selective element. A variable 
attenuator is placed in each ring to balance the cavity losses. The central wavelengths of the Fabry-Perot filter in the ring cavity 1 and 2 are $\lambda_{1}$ and $\lambda_{2}$, respectively. The SOA is biased with $250 \mathrm{~mA}$ injection current. It is shown in Ref. [4] that the roundtrip conditions of the two ring cavities can not be satisfied simultaneously due to the presence of feedback light. As a result of this the intensity of the lasing state shows periodic oscillations. The system shows bistable behavior in the sense that only one of the two lasers can lase while the other is suppressed (Fig. 3(c) and 3(d)). Switching between the two states takes place by injecting external light, at the same central wavelength of the suppressed laser, to the port 'In1'. The threshold switching power of the external light is $-15.0 \mathrm{dBm}$. The length of the ring cavity is about $10 \mathrm{~m}$ employing a fiber pig-tailed setup, which leads to a switching time of $5.0 \mu \mathrm{s}$.

When the power of the external light is below the threshold value, the system maintains the initial state. In this case, another external light input (with $3 \mathrm{dBm}$ of power) at a central wavelength of $1559.30 \mathrm{~nm}\left(\lambda_{3}\right)$ is injected into the optical flip-flop via the port 'In2. This additional input light deeply saturates the SOA so that both lasers are suppressed. In the remainder of this paper, we refer to this process as clearing of the memory. After clearing the optical flip-flop, the light injection via the port 'In2' is stopped and the light injected via the port 'In1' sets the new state of the memory. Thus the optical flip-flop starts lasing at the wavelength of the light injected via the port 'In1'.

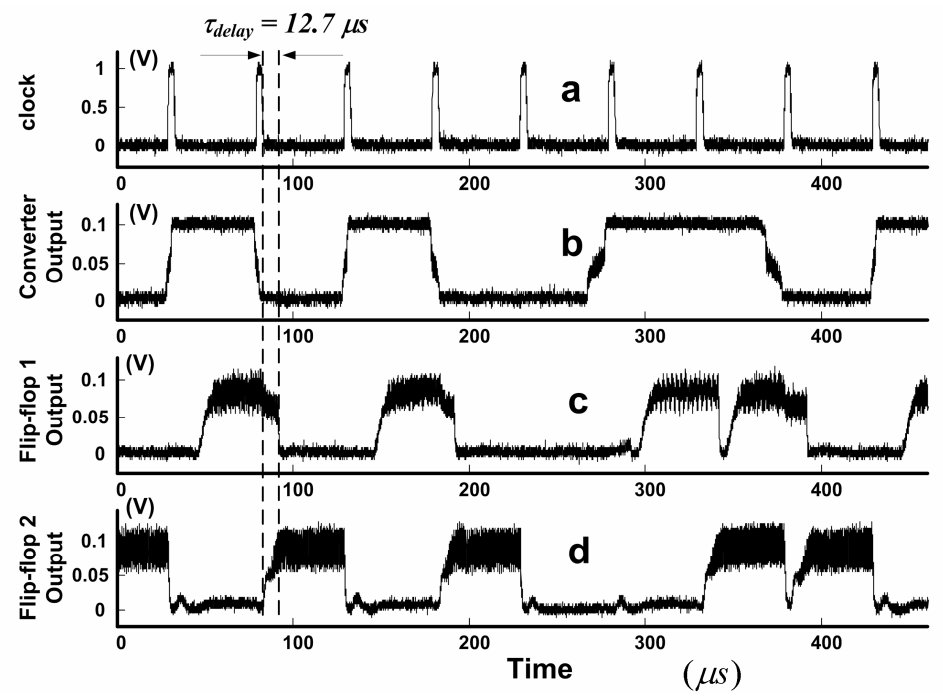

Fig. 4. Experimental results for shifting of the binary sequence 01010011 . (a): The $20 \mathrm{kHz}$ clock signal. (b) The output of the optical converter. (c) The output of optical flip-flop 1. (d) The output of optical flip-flop 2. All the signals in panels (b), (c) and (d) are filtered by a filter with a center wavelength of $\lambda_{2}(1552.92 \mathrm{~nm})$.

The optical converter combined with two cascaded optical flip-flops form the optical shift register. Figure 4 shows how the information of a binary 01010011 signal is shifted. As shown in Fig. 4(a), the $20 \mathrm{kHz}$ optical clock pulses are at the wavelength $\lambda_{3}$ and have a pulse-width of $4.0 \mu$ s (Full Width at Half Maximum). The intensity encoded input signal operates at the same frequency and is synchronized with the clock pulses. The input signal is firstly transformed into wavelength encoded signal by the optical converter, and is then injected into the port 'In1' of optical flip-flop 1, which is cascaded with optical flip-flop 2. The output of the optical converter, optical flip-flop 1 and optical flip-flop 2 are shown in Fig. 4(b), 4(c) and 4(d), respectively. In order to present the experimental results explicitly, the three outputs are filtered using a filter with a central wavelength of $\lambda_{2}$. Hence, a '0' state in Fig. 4(c) and 4(d) correspond to a lasing state at the wavelength $\lambda_{1}$ as shown in Fig. 3(c). It is shown in Fig. 4 that both optical flip-flops keep their initial states until they are cleared by the strong clock 
pulses. This is because the optical power injected into each optical flip-flop via the port 'In1' is below the threshold power and the optical flip-flops can only be cleared by a well-timed clock pulse injected via the port 'In2'. The optical power of the clock signal is split into two parts. $50 \%$ of the power is directly injected into the port 'In2' of optical flip-flop 2 to clear this memory. After $4 \mu$ s (the duration of the clock pulse), the output of optical flip-flop 1 sets the new state of optical flip-flop 2. This procedure is repeated for optical flip-flop 1 using the other $50 \%$ of the power of the clock pulse delayed by $12.7 \mu \mathrm{s}$. The new state of optical flipflop 1 is then set by the output of the optical converter which leads to the completion of the 'shift' function. All the operations take place within one clock cycle. Note that the seemingly noisy output from each flip-flop shown in Figs. 4(c) and 4(d) is due to the feedback induced oscillation of the intensity of the lasing light in each flip-flop. The oscillation frequency is typically at a few hundreds of $\mathrm{MHz}$, which is so fast compared with the $10 \mathrm{KHz}$ operation speed that the output looks like noisy. The way to improve the noise performance is to use a threshold functional device, like the optical coveter shown in Fig. 2, to smooth out the oscillation.

A shift register should not only shift information, but also maintain the state in the absence of clock pulses, which is demonstrated as follows. The data to be shifted is a repetitive binary 01010101 signal. The $20 \mathrm{kHz}$ optical clock pulses are modulated in such a way that every six consecutive optical pluses are followed by five consecutive blank cycles as shown in Fig. 5(a). The output of the optical converter, optical flip-flop 1 and optical flip-flop 2 are shown in Figs. 5(b), 5(c) and 5(d), respectively. In order to show the experimental results explicitly, the three outputs are filtered using a filter with a central wavelength of $\lambda_{2}$, Hence, a ' 0 ' state in Fig. 4(c) and 4(d) corresponds to a lasing state at the wavelength $\lambda_{1}$ as shown in Fig. 3(c). It is shown in Fig. 4 that both optical flip-flops keep their states in the absence of clock pulses, while the system starts to shift data with an injected optical clock pulse.

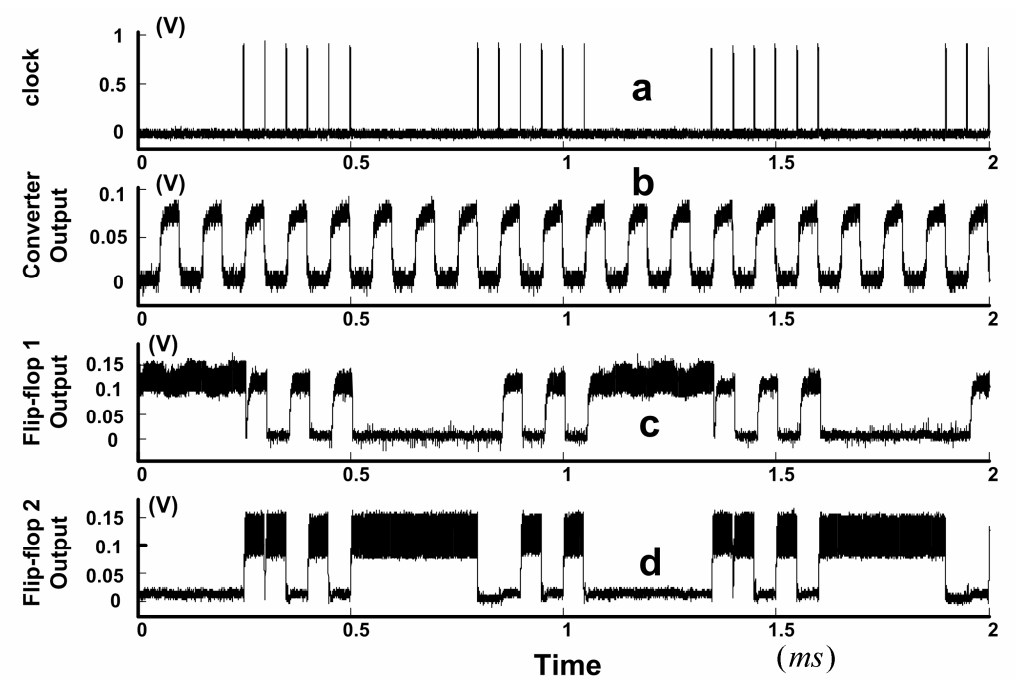

Fig. 5. Experimental results when the clock signal is modulated such that every six consecutive pluses are followed by five consecutive blank cycles. (a) The $20 \mathrm{kHz}$ clock signal. (b) The output of the optical converter. (c) The output of optical flip-flop 1. (d) The output of optical flip-flop 2. All the signals in panels (b), (c) and (d) are filtered by a filter with a center wavelength of $\lambda_{2}(1552.92 \mathrm{~nm})$.

\section{Conclusion}

An optical shift register based on serially connected optical flip-flop memories is experimentally demonstrated at an operation speed of $20 \mathrm{kHz}$. The operation speed is limited 
by the slow switching speed of the optical flip-flop memory due to $10 \mathrm{~m}$ long ring cavity made by fiber-pig-tailed components. Photonic integration would decrease the dimensions of the building blocks and increases the operation speed of the shift register.

Optical shift registers have advantages over fiber delay line. A simple delay line can only delay the signal, while an optical shift register can store data and only shift data as a response on clock pulses. Besides its potential for photonic integration, more complex functionalities like tunable shift slot and serial-to-parallel converters are feasible, since it digitally manipulates optical data at a bit-level. Furthermore, the optical shift register demonstrates that cascaded optical flip-flops can perform more sophisticated digital optical signal processing functions. In principle, the concept can also be applied using different flip-flop concepts with ultra-fast operation speed.

\section{Acknowledgments}

This work was supported by the Technology Foundation STW through the Innovation Research Incentives Scheme program.

\#7971 - \$15.00 USD

(C) 2005 OSA
Received 29 June 2005; revised 11 November 2005; accepted 11 November 2005

28 November 2005 / Vol. 13, No. 24 / OPTICS EXPRESS 9713 